\title{
Dyson-Schwinger Equations: An Instrument for Hadron Physics*
}

\author{
A. Krassnigg ${ }^{a}$ and C.D. Roberts ${ }^{\mathrm{a}, \mathrm{b}}$

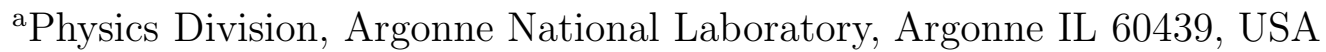 \\ ${ }^{\mathrm{b}}$ Fachbereich Physik, Universität Rostock, D-18051 Rostock, Germany
}

Dyson-Schwinger equations furnish a Poincaré covariant approach to hadron physics. They reveal that dynamical chiral symmetry breaking is tied to the long-range behaviour of the strong interaction and make predictions corroborated by modern lattice-QCD simulations. A hallmark in the contemporary use of DSEs is the existence of a nonperturbative, symmetry preserving truncation that enables the proof of exact results. The systematic error associated with the truncation's leading term has been quantified and this underpins an efficacious one-parameter model that is being employed to study meson excited states.

\section{DRESSED-QUARK PROPAGATOR}

The Dyson-Schwinger equations (DSEs) are a nonperturbative approach to studying continuum QCD [ 1, 2]. At the simplest level they provide a generating tool for perturbation theory and, because QCD is asymptotically free, this feature has the potential to materially reduce any model dependence in DSE applications. This comment is explained by noting that the long-range behaviour of the interaction between light-quarks is hitherto unknown and must therefore be modelled. Hence, the fact that a weak coupling expansion of the DSEs reproduces every diagram in perturbation theory entails that in this approach the model dependence is restricted to the infrared domain $k^{2} \lesssim 1 \mathrm{GeV}^{2}$.

The DSEs are a nonperturbative tool and can therefore be used directly to study: hadrons as bound states; the importance of dynamical chiral symmetry breaking (DCSB); and the confinement of quarks and gluons. These phenomena are all linked to the infrared behaviour of the interaction. Hence the DSEs provide a bridge between this behaviour and observables, and thereby a means by which it can be charted.

The best known DSE is the simplest: the gap equation, which describes how a fermion's propagator is modified by interactions with the medium being traversed. In QCD that equation is the DSE for the dressed-quark propagator:

$S(p)^{-1}=Z_{2}\left(i \gamma \cdot p+m_{\text {bare }}\right)+Z_{1} \int_{q}^{\Lambda} g^{2} D_{\mu \nu}(p-q) \frac{\lambda^{a}}{2} \gamma_{\mu} S(q) \Gamma_{\nu}^{a}(q ; p)$,

wherein: $D_{\mu \nu}(k)$ is the dressed-gluon propagator; $\Gamma_{\nu}^{a}(q ; p)$ is the dressed-quark-gluon vertex; $m_{\text {bare }}$ is the $\Lambda$-dependent current-quark bare mass; and $\int_{q}^{\Lambda}:=\int^{\Lambda} d^{4} q /(2 \pi)^{4}$ repre-

${ }^{*}$ CDR thanks the organisers for their hospitality and support. This work was also supported by: $F W F$ Erwin-Schrödinger-Auslandsstipendium Nr. J2233-N08; Department of Energy Nuclear Physics Division contract no. W-31-109-ENG-38; and the A.v. Humboldt Foundation via a F.W. Bessel Research Award. 
sents a translationally-invariant regularisation of the integral, with $\Lambda$ the regularisation mass-scale. In addition, $Z_{1,2}\left(\zeta^{2}, \Lambda^{2}\right)$ are the quark-gluon-vertex and quark wave function renormalisation constants, which depend on $\Lambda$ and the renormalisation point, $\zeta$, as does the mass renormalisation constant $Z_{m}\left(\zeta^{2}, \Lambda^{2}\right)=Z_{4}\left(\zeta^{2}, \Lambda^{2}\right) / Z_{2}\left(\zeta^{2}, \Lambda^{2}\right)$. The solution has the form

$S^{-1}(p)=i \gamma \cdot p A\left(p^{2}, \zeta^{2}\right)+B\left(p^{2}, \zeta^{2}\right) \equiv \frac{1}{Z\left(p^{2}, \zeta^{2}\right)}\left[i \gamma \cdot p+M\left(p^{2}\right)\right]$

where $M\left(\zeta^{2}\right) \equiv m(\zeta):=m_{\text {bare }}(\Lambda) Z_{m}^{-1}\left(\zeta^{2}, \Lambda^{2}\right)$ is the running quark mass.

The gap equation illustrates the features and flaws of each DSE. It is a nonlinear integral equation for $S(p)$ and hence can yield much-needed nonperturbative information. However, the kernel involves the two-point function $D_{\mu \nu}(k)$ and the three-point function $\Gamma_{\nu}^{a}(q ; p)$. The gap equation is therefore coupled to the DSEs these functions satisfy. Those equations in turn involve higher $n$-point functions and hence the DSEs are a tower of coupled integral equations with a tractable problem obtained only once a truncation scheme is specified. It is unsurprising that the best known truncation scheme is the weak coupling expansion, which reproduces every diagram in perturbation theory. This scheme is systematic and valuable in the analysis of large momentum transfer phenomena because QCD is asymptotically free but it precludes any possibility of obtaining nonperturbative information, which we identified as a key aspect of the DSEs.

In spite of the problem with truncation, gap equations have long been used efficaciously in obtaining nonperturbative information about many-body systems. The positive outcomes have been achieved through the simple expedient of employing a rudimentary truncation and comparing the results with observations. Naturally, agreement under these circumstances is not compelling evidence that the contributions omitted are small. However, it does justify further study, and an accumulation of good results is grounds for a concerted attempt to substantiate a reinterpretation of the truncation as the first term in a systematic and reliable approximation. That has recently been achieved [3].

The gap equation's solution has long been of interest in grappling with DCSB and typical results are depicted in Fig. 1. The infrared suppression of $Z\left(p^{2}\right)$ and enhancement of $M\left(p^{2}\right)$ evident in this figure are longstanding predictions of DSE studies [4], and in this behaviour one perceives directly the evolution of a current-quark into a constituent-quark. A critical feature is that, so long as the kernel of the gap equation has sufficient integrated strength on the infrared domain, a nonzero running quark mass is obtained even in the chiral limit. This effect is DCSB. It is impossible at any finite order of perturbation theory and also apparent in Fig. 1. The precise connection between the dressed-quark propagator and vacuum quark condensate is discussed in Ref. [ 5], and a connection with quark confinement is canvassed in Ref. [6].

The dressed-quark propagator can be calculated in lattice-regularised QCD. Results are available in the quenched truncation, and depicted in Fig. 1]are those of Ref. [7] obtained with the current-quark masses $(\zeta=19 \mathrm{GeV})$

\begin{tabular}{l|lll}
$a m_{\text {lattice }}$ & 0.018 & 0.036 & 0.072 \\
\hline$m(\zeta)(\mathrm{GeV})$ & 0.030 & 0.055 & 0.110
\end{tabular}

The precise agreement with DSE results is not accidental. (NB. The chiral limit discrepancy points to the inadequacy of a linear extrapolation of lattice data to this limit [6].) 

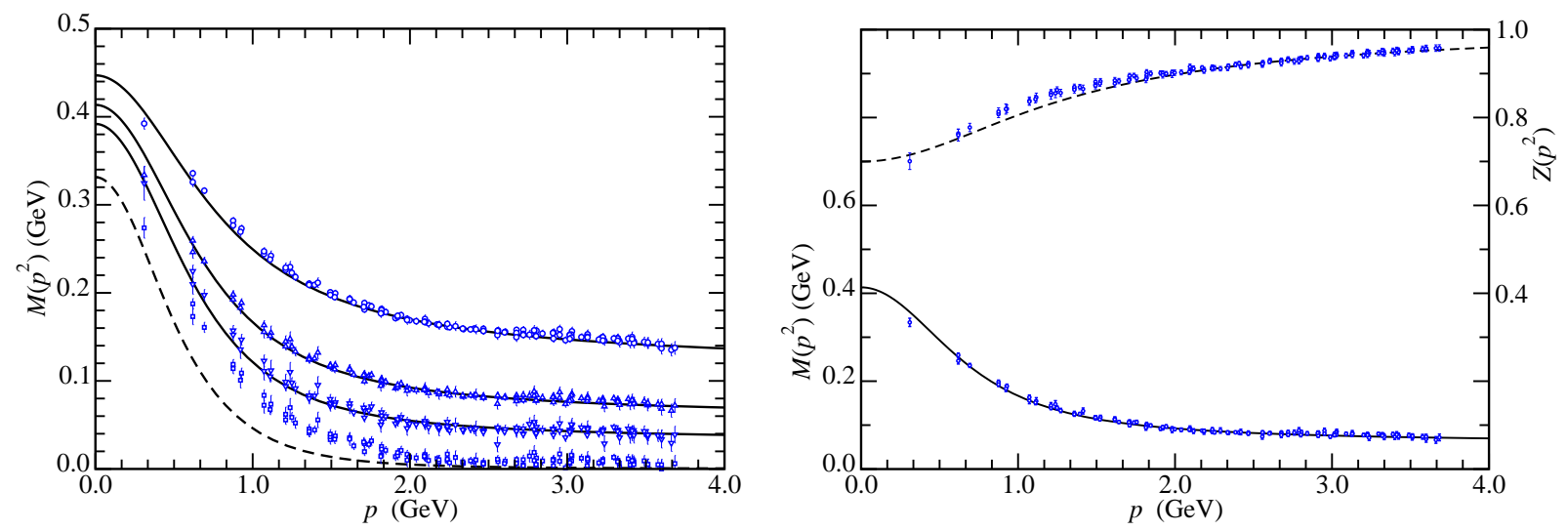

Figure 1. Left Panel - Dashed-curve: gap equation's solution in the chiral limit; solid curves: solutions for $M\left(p^{2}\right)$ obtained using $m(\zeta)$ in Eq. (3). (From Ref. [6].) Data, upper three sets: lattice results for $M\left(p^{2}\right)$ in $\mathrm{GeV}$ at $a m$ values in Eq. (3); lower points (boxes): linear extrapolation of these results [7] to am $=0$. Right Panel-Dashed curve, $Z\left(p^{2}\right)$, and solid curve, $M\left(p^{2}\right)$ calculated from the gap equation with $m(\zeta)=55 \mathrm{MeV}$ [ 6]. Data, quenched lattice-QCD results for $M\left(p^{2}\right)$ and $Z\left(p^{2}\right)$ obtained with $a m=0.036$ [ [7].

The essential agreement between lattice results and DSE predictions was highlighted in Refs. [ 8, 9] but Ref. [ 6] pursued a different goal. Only recently has reliable information about the gap equation's kernel at infrared momenta begun to emerge, in the continuum [10] and on the lattice [11]. Reference [6] therefore employed an Ansatz for the infrared behaviour of the gap equation's kernel in order to demonstrate that it is possible to correlate lattice results for the gluon and quark Schwinger functions via QCD's gap equation. This required the gap equation's kernel to exhibit infrared enhancement over and above that observed in the gluon propagator alone, which could be attributed to an amplification of the dressed-quark-gluon vertex whose magnitude is consistent with that observed in quenched lattice estimates of this three-point function [ 12].

\section{HADRONS}

It is evident that reliable knowledge of QCD's two-point functions (the propagators for QCD's elementary excitations) is available. Direct comparison with experiment requires an equally good understanding of bound states. Progress here has required the evolution of an understanding of the intimate connection between symmetries and DSE truncation schemes. This is well illustrated by considering the pion, whose properties are profoundly connected with DCSB. Indeed, the correct understanding of pion observables requires an approach to contain a well-defined and valid chiral limit.

Chiral symmetry and its breaking are expressed through the axial-vector Ward-Takahashi identity

$P_{\mu} \Gamma_{5 \mu}^{j}(k ; P)=\mathcal{S}^{-1}\left(k_{+}\right) i \gamma_{5} \frac{\tau^{j}}{2}+i \gamma_{5} \frac{\tau^{j}}{2} \mathcal{S}^{-1}\left(k_{-}\right)-i \mathcal{M}(\zeta) \Gamma_{5}^{j}(k ; P)-\Gamma_{5}^{j}(k ; P) i \mathcal{M}(\zeta)$,

where $k_{ \pm}=k \pm P / 2$, and $\left\{\tau^{j}, j=1,2,3\right\}$ are the Pauli matrices as herein we focus on $S U(2)$-flavour. This identity connects the axial-vector vertex: $\Gamma_{5 \mu}^{j}(k ; P), P$ is the 


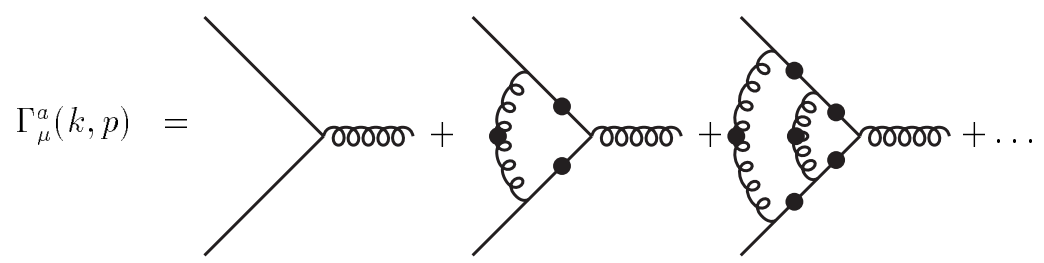

Figure 2. Planar dressed-quark-gluon vertex obtained by neglecting contributions associated with explicit gluon self-interactions. Solid circles indicate fully dressed propagators. The quark-gluon vertices are not dressed.

total momentum, with the dressed quark propagator: $\mathcal{S}=\operatorname{diag}\left[S_{u}, S_{d}\right]$, the pseudoscalar vertex: $\Gamma_{5}^{j}(k ; P)$, and the current-quark mass matrix: $\mathcal{M}(\zeta)=\operatorname{diag}\left[m_{u}(\zeta), m_{d}(\zeta)\right]$. The propagator satisfies the gap equation but the vertices are determined by inhomogeneous Bethe-Salpeter equations; e.g.,

$$
\left[\Gamma_{5 \mu}^{j}(k ; P)\right]_{t u}=Z_{2}\left[\gamma_{5} \gamma_{\mu} \frac{\tau^{j}}{2}\right]_{t u}+\int_{q}^{\Lambda}\left[\mathcal{S}\left(q_{+}\right) \Gamma_{5 \mu}^{j}(q ; P) \mathcal{S}\left(q_{-}\right)\right]_{s r} K_{t u}^{r s}(q, k ; P),
$$

wherein $K(q, k ; P)$ is the dressed-quark-antiquark scattering kernel. The importance of DCSB entails that any truncation useful in understanding low energy phenomena must be nonperturbative and preserve Eq. (41) without fine tuning. This constraint requires an intimate connection between $K(q, k ; P)$ and the gap equation's kernel.

One systematic and nonperturbative truncation scheme has been identified that explicates this connection, and hence preserves QCD's global symmetries [ [3]. It is a dressedloop expansion of the dressed-quark-gluon vertices that appear in the half-amputated dressed-quark-antiquark scattering matrix: $S^{2} K$. In this scheme, as in perturbation theory, it is impossible, in general, to obtain complete closed-form expressions for the kernels of the gap and Bethe-Salpeter equations. However, for the planar dressed-quark-gluon vertex depicted in Fig. 2. closed forms can be obtained and a number of significant features illustrated [3] when one uses the following model for the dressed-gluon line [13]

$g^{2} D_{\mu \nu}(k)=\left[\delta_{\mu \nu}-\frac{k_{\mu} k_{\nu}}{k^{2}}\right](2 \pi)^{4} \mathcal{G}^{2} \delta^{4}(k)$,

where $\mathcal{G}$ sets the model's mass-scale. This form has many positive attributes in common with the class of renormalisation-group-improved models and furthermore its particular momentum-dependence works to advantage in reducing integral equations to characterpreserving algebraic equations.

It is a general result [3] that with any vertex whose diagrammatic content is explicitly known; e.g., Fig.2 it is always possible to construct a unique Bethe-Salpeter kernel which ensures the Ward-Takahashi identities are automatically fulfilled. That kernel is necessarily non-planar. This becomes transparent with the model in Eq. (6) , using which the gap equation obtained with the vertex depicted in Fig.2 reduces to an algebraic equation, irrespective of the number of dressed-gluon rungs that are retained, and the same is true of the Bethe-Salpeter equations in every channel: pseudoscalar, vector, etc. 
Table 1

$\pi$ and $\rho$ meson masses obtained with $\mathcal{G}=0.48 \mathrm{GeV}$. (Dimensioned quantities in $\mathrm{GeV}$.) $n$ is the number of dressed-gluon rungs retained in the planar vertex, see Fig. 2, and hence the order of the vertex-consistent Bethe-Salpeter kernel.

\begin{tabular}{l|cccc}
\hline & $M_{H}^{n=0}$ & $M_{H}^{n=1}$ & $M_{H}^{n=2}$ & $M_{H}^{n=\infty}$ \\
\hline$\pi, m=0$ & 0 & 0 & 0 & 0 \\
$\pi, m=0.011$ & 0.152 & 0.152 & 0.152 & 0.152 \\
\hline$\rho, m=0$ & 0.678 & 0.745 & 0.754 & 0.754 \\
$\rho, m=0.011$ & 0.695 & 0.762 & 0.770 & 0.770 \\
\hline
\end{tabular}

Results for the $\pi$ and $\rho$ are illustrated in Table 1. It is evident that, irrespective of the order of the truncation; i.e., the number of dressed gluon rungs in the quark-gluon vertex, the pion is massless in the chiral limit. (NB. This pion is composed of heavy dressed-quarks, as is evident in the calculated scale of the dynamically generated dressedquark mass function: $M(0) \approx 0.5 \mathrm{GeV}$; viz., dynamically generated constituent-quarks compose the pion.) The masslessness of the $\pi$ is a model-independent consequence of the consistency between the Bethe-Salpeter kernel and the kernel in the gap equation. Furthermore, the bulk of the $\rho-\pi$ mass splitting is present in the chiral limit and with the simplest ( $n=0$; i.e., rainbow-ladder) kernel, which makes plain that this mass difference is driven by the DCSB mechanism: it is not the result of a finely adjusted hyperfine interaction. Finally, the quantitative effect of improving on the rainbow-ladder truncation; viz., including more dressed-gluon rungs in the gap equation's kernel and consistently improving the kernel in the Bethe-Salpeter kernel, is a $10 \%$ correction to the vector meson mass. Simply including the first correction $(n=1$; i.e., retaining the first two diagrams in Fig. (2) yields a vector meson mass that differs from the fully resummed result by $\lesssim 1 \%$. The rainbow-ladder truncation is clearly accurate in these channels.

\section{AB INITIO CALCULATIONS}

The leading-order term in the truncation scheme described above is the renormalisationgroup-improved rainbow-ladder truncation, which as we have just seen is reliable for flavour nonsinglet pseudoscalar and vector mesons. This truncation has been refined and exploited by Maris and Tandy in a series of articles ${ }^{1}$ via a one-parameter model for the behaviour of the dressed-quark-quark interaction:

$\frac{\alpha\left(Q^{2}\right)}{Q^{2}}=\frac{\pi}{\omega^{6}} D Q^{2} \mathrm{e}^{-Q^{2} / \omega^{2}}+\frac{\pi \gamma_{m}}{\frac{1}{2} \ln \left[\tau+\left(1+Q^{2} / \Lambda_{\mathrm{QCD}}^{2}\right)^{2}\right]} \mathcal{F}\left(Q^{2}\right)$

wherein $\mathcal{F}\left(Q^{2}\right)=\left[1-\exp \left(-Q^{2} /\left[4 m_{t}^{2}\right]\right)\right] / Q^{2}, m_{t}=0.5 \mathrm{GeV} ; \tau=\mathrm{e}^{2}-1 ; \gamma_{m}=12 / 25$; and $\Lambda_{\mathrm{QCD}}=0.234 \mathrm{GeV}$. This simple form expresses the interaction as a sum: the second term ensures that perturbative behaviour is preserved at short-range; and the first makes provision for enhancement at long-range. The true parameters in Eq. (77) are $D$ and $\omega$,

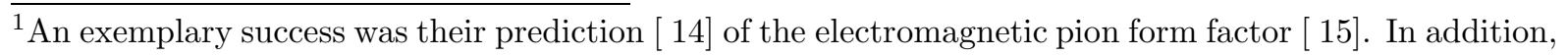
see Ref. [16] and references therein, and the review of their substantial contributions in Ref. [2]. 
which together determine the integrated infrared strength of the rainbow-ladder kernel. However, they are not independent: in fitting to a selection of observables, a change in one is compensated by altering the other; e.g., on the domain $\omega \in[0.3,0.5] \mathrm{GeV}$, the fitted observables are approximately constant along the trajectory $[8] \omega D=(0.72 \mathrm{GeV})^{3}$. Hence Eq. (7) is a one-parameter model. This correlation: a reduction in $D$ compensating an increase in $\omega$, ensures a fixed value of the integrated infrared strength. It also defines a single dressed-glue mass-scale, $m_{g}=720 \mathrm{MeV}$, which characterises infrared gluodynamics.

A new application is the calculation of properties of meson excited states, which is a first step in seeking exotics and hybrids. As an example, it is a general feature of QCD that the axial-vector vertex, obtained via Eq. (5), exhibits a pole whenever $P^{2}=-m_{\pi_{n}}^{2}$, where $m_{\pi_{n}}$ is the mass of any pseudoscalar $u$ - $d$ meson; viz.,

$$
\left.\Gamma_{5 \mu}^{j}(k ; P)\right|_{P^{2}+m_{\pi_{n}}^{2} \approx 0}=\text { regular terms }+\frac{f_{\pi_{n}} P_{\mu}}{P^{2}+m_{\pi_{n}}^{2}} \Gamma_{\pi_{n}}^{j}(k ; P),
$$

with $\Gamma_{\pi_{n}}^{j}(k ; P)$ being the $0^{-+}$bound state's Bethe-Salpeter amplitude:

$$
\begin{aligned}
\Gamma_{\pi_{n}}^{j}(k ; P)= & \tau^{j} \gamma_{5}\left[i E_{\pi_{n}}(k ; P)+\gamma \cdot P F_{\pi_{n}}(k ; P)\right. \\
& \left.+\gamma \cdot k k \cdot P G_{\pi_{n}}(k ; P)+\sigma_{\mu \nu} k_{\mu} P_{\nu} H_{\pi_{n}}(k ; P)\right],
\end{aligned}
$$

and $f_{\pi_{n}}$, its leptonic decay constant

$f_{\pi_{n}} \delta^{i j} P_{\mu}=Z_{2} \operatorname{tr} \int_{q}^{\Lambda} \frac{1}{2} \tau^{i} \gamma_{5} \gamma_{\mu} \mathcal{S}\left(q_{+}\right) \Gamma_{\pi_{n}}^{j}(q ; P) \mathcal{S}\left(q_{-}\right)$

where the trace is over colour, flavour and spinor indices. The lowest mass pole contribution, denoted by $n=0$, is the ground state pion, which receives the spectroscopic assignment $N^{2 s+1} L_{J}=1{ }^{1} S_{0}$ in the naive $q \bar{q}$ quark model. The $n \geq 1$ pseudoscalar meson poles correspond to those excited states of the pion that would receive the spectroscopic assignments $(n+1){ }^{1} S_{0}$ in the quark model.

The pseudoscalar vertex, $\Gamma_{5}^{j}(k ; P)$, which appears in Eq. (4I), also exhibits such a pole:

$$
\begin{aligned}
& \left.i \Gamma_{5}^{j}(k ; P)\right|_{P^{2}+m_{\pi_{n}}^{2} \approx 0}=\text { regular terms }+\frac{\rho_{\pi_{n}}}{P^{2}+m_{\pi_{n}}^{2}} \Gamma_{\pi_{n}}^{j}(k ; P), \\
& i \rho_{\pi_{n}}(\zeta) \delta^{i j}=Z_{4} \operatorname{tr} \int_{q}^{\Lambda} \frac{1}{2} \tau^{i} \gamma_{5} \mathcal{S}\left(q_{+}\right) \Gamma_{\pi_{n}}^{j}(q ; P) \mathcal{S}\left(q_{-}\right) .
\end{aligned}
$$

In QCD it therefore follows as a necessary consequence of chiral symmetry and its dynamical breaking that for any pseudoscalar $u$ - $d$ meson [17]

$f_{\pi_{n}} m_{\pi_{n}}^{2}=\left[m_{u}(\zeta)+m_{d}(\zeta)\right] \rho_{\pi_{n}}(\zeta)$.

(The generalisation to $S U\left(N_{f}\right)$-flavour is straightforward, following Ref. [ 18.) The socalled Gell-Mann-Oakes-Renner relation for the ground state pion appears as a corollary of Eq. (13) [19], and another important corollary of Eq. (13), valid for pseudoscalar mesons containing at least one heavy-quark, is described in Ref. [20]. In addition, the proof of Eq. (13) establishes [17] that in the chiral limit

$f_{\pi_{n \neq 0}}^{0}:=\lim _{m \rightarrow 0} f_{\pi_{n \neq 0}}=0$; 
i.e., in the chiral limit the leptonic decay constant vanishes for every pseudoscalar meson except the pion. This exact result is a constraint on models and methods used to search for exotics and hybrids.

The rainbow-ladder truncation obtained with the interaction of Eq. (17) provides an excellent description of ground state pseudoscalar mesons [ 2]. Nevertheless, the longrange part of this interaction expresses a model and its veracity can be tested further by studying excited pseudoscalar mesons. We have begun this process, focusing on the $n=1$ pseudoscalar meson ( $2{ }^{1} S_{0}$ in the quark model).

In our studies to date we have only retained the $i \gamma_{5} E_{\pi_{1}}(k ; P)$ piece in Eq. (9)). Employing this expedient for the ground state pion leads to a $25 \%$ underestimate of $m_{\pi_{0}}$. Moreover, in neglecting the pseudovector components, we omit those terms which in the pion's rest frame signal the presence of quark orbital angular momentum. In QCD they are necessarily nonzero [19, 21].

To determine $m_{\pi_{n=1}}$ we first solved a rainbow truncation of the gap equation; viz.,

$S(p)^{-1}=Z_{2}\left(i \gamma \cdot p+m_{\text {bare }}\right)+\int_{q}^{\Lambda} 4 \pi \alpha\left((p-q)^{2}\right) D_{\mu \nu}^{\text {free }}(p-q) \frac{\lambda^{a}}{2} \gamma_{\mu} S(q) \frac{\lambda^{a}}{2} \gamma_{\nu}$

where $D_{\mu \nu}^{\text {free }}(k)$ is the undressed gauge boson propagator. This allows one to complete the specification of the ladder-truncation Bethe-Salpeter equation's kernel:

$\Gamma_{\pi_{n}}(k ; P)+\int_{q}^{\Lambda} 4 \pi \alpha\left((k-q)^{2}\right) D_{\mu \nu}^{\mathrm{free}}(k-q) \frac{\lambda^{a}}{2} \gamma_{\mu} \mathcal{S}\left(q_{+}\right) \Gamma_{\pi_{n}}(q ; P) \mathcal{S}\left(q_{-}\right) \frac{\lambda^{a}}{2} \gamma_{\nu}=0$,

which can be solved as described in Ref. [17] to obtain the mass of the ground state and excited pseudoscalar mesons.

One should note that in relativistic quantum field theory the scalar functions in Eq. (9) depend on two variables: $k^{2}, k \cdot P$. In quantum mechanics $k \cdot P \equiv 0$, of course, because of "on-shell" constraints on the constituents, and this degree of freedom is absent. As a matter of practice, we solve Eq. (16) by using a Chebyshev decomposition; i.e., we write ${ }^{2}$

$E_{\pi_{n}}\left(k^{2}, k \cdot P ; P^{2}\right)=\sum_{i=0,2,4, \ldots}^{N_{\max }}{ }^{i} E_{\pi_{n}}\left(k^{2} ; P^{2}\right) U_{i}(\cos \beta)$,

where $\left\{U_{i}(x) ; i=0, \ldots, \infty\right\}$ are Chebyshev polynomials of the second kind, and increase $N_{\max }$ until $m_{\pi_{n}}$ stabilises. This procedure has the merit of limiting the amount of computer memory required. It also converges very rapidly for the ground state pion, with only the leading Chebyshev moment being required [18], as apparent in Fig.3]

We have obtained the mass and amplitude for the ground state pion, using the complete expression in Eq. (9), and found: $f_{\pi_{0}}=0.092 \mathrm{GeV} ; m_{\pi_{0}}=0.14 \mathrm{GeV} ; \rho_{\pi_{0}}=(0.81 \mathrm{GeV})^{2}$, at a current-quark mass $m_{d}(1 \mathrm{GeV})=m_{u}(1 \mathrm{GeV})=5.5 \mathrm{MeV}$, reproducing the results in Ref. [14]. We can report in addition that using solely the $i \gamma_{5} E_{\pi_{1}}(k ; P)$ term in Eq. (9)

$m_{\pi_{1}}=1.1 \mathrm{GeV}$,

cf. $m_{\pi_{1}}^{\text {expt. }}=1.3 \pm 0.1 \mathrm{GeV}$. In omitting the pseudoscalar meson's pseudovector components one violates Eq. (41) [18] and hence we are not yet in a position to verify that Eq. (14) is

\footnotetext{
${ }^{2}$ The Bethe-Salpeter amplitude for charge conjugation eigenstates is even under $k \cdot P \rightarrow-k \cdot P$ and hence only even Chebyshev moments contribute.
} 


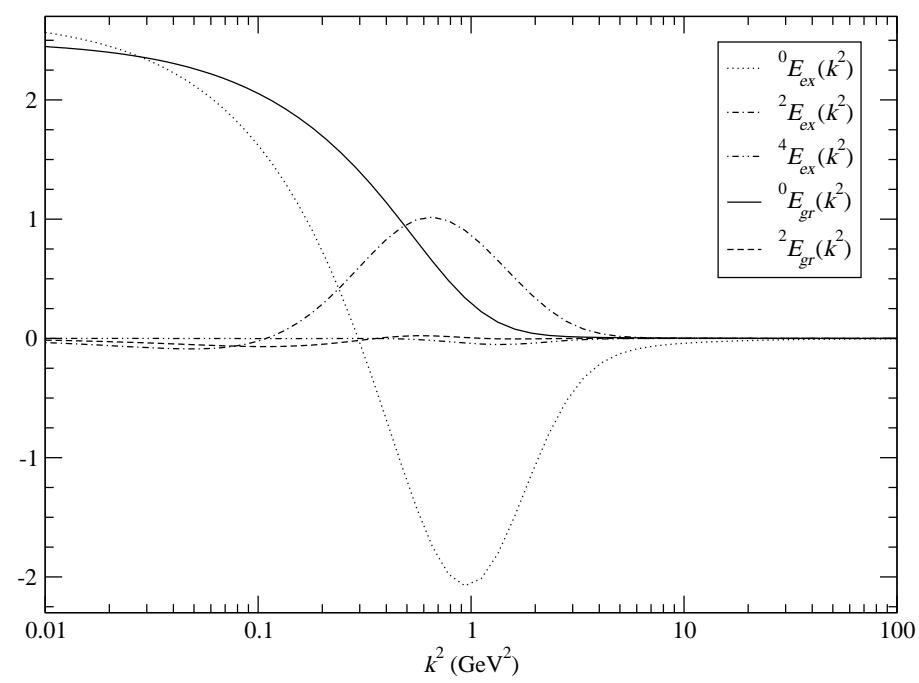

Figure 3. Chebyshev moments, Eq. (17), of $E_{\pi_{0}}(k ; P) \equiv E_{g r}(k ; P)$ and $E_{\pi_{1}}(k ; P) \equiv$ $E_{e x}(k ; P)$. The leading moment is sufficient to accurately represent the ground state while a veracious description of the first excited state requires inclusion of the second moment. Note the appearance of a single node in each of the first excited state's Chebyshev moments. (The amplitudes are canonically normalised.)

preserved in the rainbow-ladder truncation. Nevertheless, we estimate $f_{\pi_{n=1}} \leq 1.5 \mathrm{MeV}$ at the physical current-quark mass [17].

We plot the leading Chebyshev moments of $E_{\pi_{1}}(k ; P)$ in Fig. 3. wherefrom it appears that the first two moments are sufficient for an accurate representation of this amplitude but confirmation must await the inclusion of the pseudovector components. It is worth remarking that the moments of $E_{\pi_{1}}(k ; P)$ each possess a single node and exhibit tails that extend to larger values of $k^{2}$ than their analogue in the ground state. These observations are redolent of those one could make in comparing Fourier transforms of the radial wave functions for bound states in a potential well. This fact emphasises the intuitive character of Bethe-Salpeter amplitudes.

\section{EPILOGUE}

There are many additional applications of interest to this community, among them: the $a b$ initio calculation of electroweak and transition form factors for other mesons, and $\pi \pi$ scattering [ 22]; and a calculation of the pion's valence-quark distribution function, whose discrepancy with extant data raises difficult questions [23]. A pressing contemporary challenge is the extension of the framework to the calculation of baryon observables, aspects of which are beginning to be understood [24]. 


\section{REFERENCES}

1. C. D. Roberts and S. M. Schmidt, Prog. Part. Nucl. Phys. 45 (2000) S1; R. Alkofer and L.v. Smekal, Phys. Rept. 353 (2001) 281.

2. P. Maris and C. D. Roberts, Int. J. Mod. Phys. E 12 (2003) 297.

3. A. Bender, C.D. Roberts and L. von Smekal, Phys. Lett. B 380 (1996) 7; A. Bender, W. Detmold, C.D. Roberts and A.W. Thomas, Phys. Rev. C 65 (2002) 065203.

4. C.D. Roberts and A.G. Williams, Prog. Part. Nucl. Phys. 33 (1994) 477.

5. K. Langfeld, H. Markum, R. Pullirsch, C.D. Roberts and S.M. Schmidt, Phys. Rev. C 67 (2003) 065206.

6. M.S. Bhagwat, M.A. Pichowsky, C.D. Roberts and P.C. Tandy, Phys. Rev. C 68 (2003) 015203.

7. P.O. Bowman, U.M. Heller, D.B. Leinweber and A.G. Williams, "Modelling the quark propagator," hep-lat/0209129.

8. P. Maris, A. Raya, C.D. Roberts and S.M. Schmidt, "Facets of confinement and dynamical chiral symmetry breaking," nucl-th/0208071;

9. P.C. Tandy, Prog. Part. Nucl. Phys. 50 (2003) 305.

10. C.S. Fischer and R. Alkofer, Phys. Rev. D 67 (2003) 094020; and references therein.

11. P.O. Bowman, U.M. Heller, D.B. Leinweber and A.G. Williams, Phys. Rev. D 66 (2002) 074505; and references therein.

12. J.I. Skullerud, P.O. Bowman, A. Kızılersü, D.B. Leinweber and A.G. Williams, JHEP 0304 (2003) 047.

13. H.J. Munczek and A.M. Nemirovsky, Phys. Rev. D 28 (1983) 181.

14. P. Maris and P.C. Tandy, Phys. Rev. C 61 (2000) 045202; ibid., C 62 (2000) 055204.

15. J. Volmer et al. [JLab $F_{\pi}$ Collaboration], Phys. Rev. Lett. 86 (2001) 1713.

16. D. Jarecke, P. Maris and P.C. Tandy, Phys. Rev. C 67 (2003) 035202.

17. A. Krassnigg and C.D. Roberts, "DSEs, the pion, and related matters," nucl-th/0308039.

18. P. Maris and C.D. Roberts, Phys. Rev. C 56 (1997) 3369.

19. P. Maris, C.D. Roberts and P.C. Tandy, Phys. Lett. B 420 (1998) 267.

20. M.A. Ivanov, Yu.L. Kalinovsky and C.D. Roberts, Phys. Rev. D 60 (1999) 034018.

21. P. Maris and C.D. Roberts, Phys. Rev. C 58 (1998) 3659.

22. P. Bicudo, S. Cotanch, F. Llanes-Estrada, P. Maris, E. Ribeiro and A. Szczepaniak, Phys. Rev. D 65 (2002) 076008; P. Bicudo, Phys. Rev. C 67 (2003) 035201.

23. R. J. Holt and P. E. Reimer, AIP Conf. Proc. 588, 234 (2001); C.D. Roberts, Nucl. Phys. Proc. Suppl. 108 (2002) 227.

24. J.C.R. Bloch, A. Krassnigg and C.D. Roberts, "Regarding proton form factors," nucl-th/0306059, and references therein. 